\title{
The measurement and modeling of the levitation force between single grain YBCO bulk superconductors and permanent magnets
}

\author{
Antonio Morandi, Senior Member, IEEE, Massimo Fabbri, Pier Luigi Ribani, Anthony Dennis, \\ John Durrell, Yunhua Shi and David Cardwell
}

\begin{abstract}
The levitation force between a single grain YBCO cylindrical bulk superconductor and a permanent magnet is investigated. The force measured both in zero field cooling and field cooling condition in the temperature range $20 \mathrm{~K}-80 \mathrm{~K}$ is reported. The dependence of peak levitation force and power dissipation on the temperature is analyzed and the saturation of the peak force with increasing the critical current of the material is pointed out. A numerical analysis is also carried out for understanding the electrodynamics of the levitation mechanism. Good reproduction of the measured data is obtained and relevant observed phenomena, such as hysteresis of the levitation cycle at high temperature and saturation of the peak force at low temperature, are explained by means of the model. Characterization of the material in terms of critical current density in the field range $0 \mathrm{~T}-0.5 \mathrm{~T}$ is also obtained by means of the numerical model by means of the fitting of the measured data.
\end{abstract}

Index Terms - Magnetic levitation, bulk superconductor, numerical modelling, material characterization

\section{INTRODUCTION}

B ULK single grain (RE)BCO superconductor samples, with size up to several centimeters, can now be fabricated by means of the melt-growth method [1]-[5]. The large size, combined with the high critical current density at high magnetic field, allows the development of devices with unachieved performance in terms of trapped field [5] and levitation force, able to operate in the temperature range of $20 \mathrm{~K}-65 \mathrm{~K}$. These devices have the potential to produce a substantial impact in the transportation and the energy sectors [6]. Specifically, levitation systems based on bulk high temperature superconductors, can be exploited in variety of applications, including rail transportation, motors/generators and flywheel energy storage [7]-[9].

Characterization is essential for the development of levitating systems based on (RE)BCO bulks. Furthermore, numerical modeling is required for understanding the electrodynamics of the observed levitation phenomena and for predicting and optimizing the performance of systems of practical interest [10]-[20]. Numerical modeling also provides a way for

Automatically generated dates of receipt and acceptance will be placed here; authors do not produce these dates. (Corresponding author: Antonio Morandi)

A. Morandi, M. Fabbri and P. L. Ribani are with the University of Bologna - Department of Electrical, Electronic and Information Engineering, 40136 Bologna, Italy (e-mail: antonio.morandi@unibo.it, massimo.fabbri@unibo.it, pierluigi.ribani@unibo.it). deducing, by the fitting of the experimental data, the critical current density of the material based on the measured levitation force [20]. In this paper, the levitation force between a permanent magnet (PM) and a single grain YBCO sample is investigated experimentally and numerically in the temperature range $30 \mathrm{~K}-80 \mathrm{~K}$. The paper is organized as follows: the experimental apparatus used for the measurement of the levitation force in field cooling and zero field cooling conditions is described in section II. Experimental results are presented and discussed in section III; the dependence on the temperature and the saturation of the levitation force at low temperature are pointed out. The numerical model is presented in section IV. Numerical results are discussed and compared with experiments in section V. Values of critical current density at different temperature and magnetic field, deduced by the fitting of the experimental data, are also reported.

\section{THE LEVITATION FACILITY}

An experimental facility for the measurement of the levitation forces between permanent magnets and large superconductor samples has been established at the Laboratory of Magnet Engineering and Applied Superconductivity (LIMSA) of the University of Bologna. The facility, originally developed by EDISON SpA [14]-[15], consists of a vacuum chamber surrounding a copper plate connected to the second stage of a Sumitomo RDK 415D cryocooler. A schematic of the facility is shown in Fig. 1. The main characteristics are resumed in Table I. The superconductor is in thermal contact with the copper plate (which also acts as mechanical support), and can reach a temperature of $20 \mathrm{~K}$ without the need of additional thermal shields. Temperature is measured by means of two diodes (LakeShore, type D-670-SD-4L) mounted onto the bottom side of the plate. Cooling of the superconductor at $20 \mathrm{~K}$ can be obtained in about 2 hours. Lower temperatures down to $10 \mathrm{~K}$ can be reached by adding multilayer insulation onto the superconductor sample. Superconductor bulks with maximum size up to $200 \mathrm{~mm}$ can be cooled and tested by means of the facility. The temperature of the superconductor can be

Anthony Dennis, John Durrell, Yunhua Shi and David Cardwell, are with University of Cambridge - Department of Engineering, CB2 1P Cambridge, UK (ad466@cam.ac.uk, john.durrell@eng.cam.ac.uk, ys206@cam.ac.uk, dc135@cam.ac.uk) 
controlled in the range $10 \mathrm{~K}-90 \mathrm{~K}$ by means of four heaters with feedback control mounted directly onto the copper plate. The facility is equipped with a mechanical apparatus able to drive a permanent magnet (or an arrangement of permanent magnets) along the vertical (z) and the horizontal (x) axes of measurement, and with a pair of strain gauges to measure the forces along $\mathrm{z}$ and $\mathrm{x}$. Both the force sensors are able to measure a maximum force of up to $500 \mathrm{~N}$. Mechanical apparatus was preliminary calibrated both for what concern the acquisition of the force and the acquisition of the position of the PM. The Maximum excursion of the PM along the vertical and the horizontal direction is $55.9 \mathrm{~mm}$. It is important to point out that no information is available concerning the temperature of the PM, which may impact the magnetization, when the superconductor is cooled down in the range $10 \mathrm{~K}-90 \mathrm{~K}$. However, as the PM is connected to the room temperature stepping motors by means of a large aluminum arm, its temperature should not differ very much from the ambient one. Experimental monitoring of the temperature of the PM during the experiments will be the aim of a future extension of the facility.

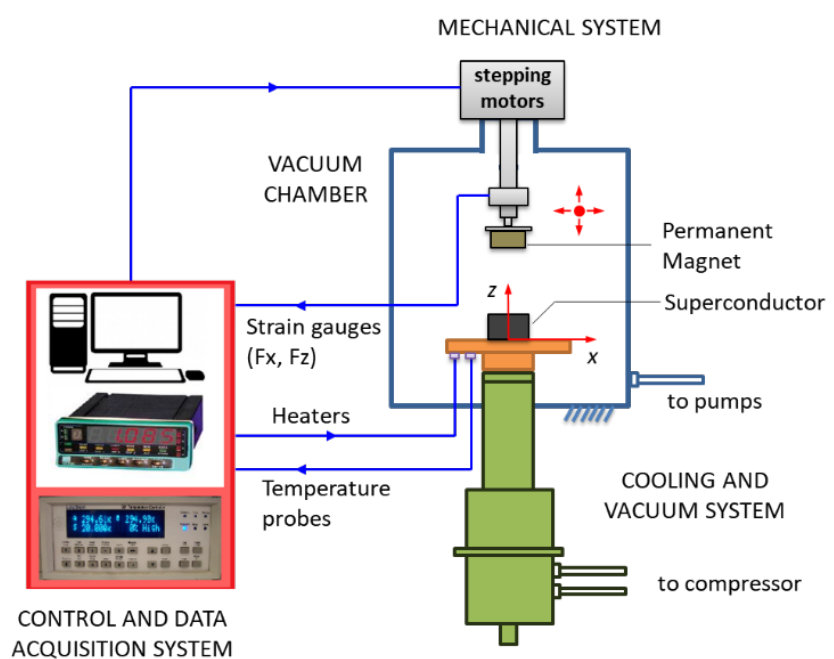

Fig. 1. Schematic of the Levitation facility

TABLE I

MAIN CHARACTERISTICS OF THE LEVITATION FACILITY

\begin{tabular}{lc}
\hline \hline Operating temperature range & $20 \mathrm{~K}-90 \mathrm{~K}$ \\
Maximum excursion of the PM $(\mathrm{x}, \mathrm{z})$ & $55.9 \mathrm{~mm}$ \\
Minimum step $(\mathrm{x}, \mathrm{z})$ & $0.1 \mathrm{~mm}$ \\
Maximum velocity & $60 \mathrm{~mm} / \mathrm{s}$ \\
Maximum measurable force (x,z) & $500 \mathrm{~N}$ \\
Max size of sample & $200 \mathrm{~mm}$ \\
\hline \hline
\end{tabular}

\section{EXPERIMENTAL RESULTS AND DISCUSSION}

\section{A. Experimental set-up}

The levitation force between a permanent magnet and a single grain YBCO sample was investigated experimentally within the temperature range $30 \mathrm{~K}-85 \mathrm{~K}$ by means of the facility. The sample, a cylinder with $25.2 \mathrm{~mm}$ in diameter and $18 \mathrm{~mm}$ thickness, was fabricated by means of the top-seeded, melt-growth (TSMG) method by the Bulk Superconductor Group in Cambridge. A SmCo cylinder with $25 \mathrm{~mm}$ in diameter and $18 \mathrm{~mm}$ thickness was used as a permanent magnet. The measured value of the axial remanent field at the center of the magnet's surface is $498 \mathrm{mT}$. More information about the field produced by the PM is reported in section IV.B. The PM and the superconductor were mounted coaxially as shown in Fig. 2. Only vertical movements of the PM (parallel to the axis) were applied, that is, axial symmetry was maintained all over the experiments. The distance $d$ between the top surface of the YBCO bulk and the bottom surface of the PM was used to indicate the relative position of the two objects during the experiments. Cyclic movement of the PM from an initial distance $d_{\mathrm{i}}$ to a final distance $d_{\mathrm{f}}$ and then back to the initial distance $d_{\mathrm{i}}$ was applied. Four consecutive cycles were performed at each temperature. Both zero field cooling experiments and field cooling experiments were carried out. In ZFC experiments the magnet was first placed at the maximum possible distance from the bulk while its temperature was above $T_{\mathrm{c}}$; the bulk was then cooled at the desired measurement temperature and after this the PM was moved toward the bulk and back again for four cycles. In FC experiments the bulk was first placed in the vicinity of the superconductor while this was kept above $T_{\mathrm{c}}$; the bulk was then cooled at the desired temperature and the PM was moved away from the bulk and back again for four times. Initial and final distance used for zero field cooling and field cooling experiments are reported in Fig. 2. The larger minimum gap used in the case of FC experiments $(1.3 \mathrm{~mm}$ vs $0.1 \mathrm{~mm})$ is due to the fact that, as attractive levitation force is obtained during the removal of the magnet, an aluminum strip with $1.2 \mathrm{~mm}$ thickness was applied on the top of the superconductor in order to avoid detachment from the copper disk and preserve good thermal contact. Both for ZFC and $\mathrm{FC}$ experiments the sample was warmed above Tc between two cycles of measurements at different temperature, so as to return in the virgin state.

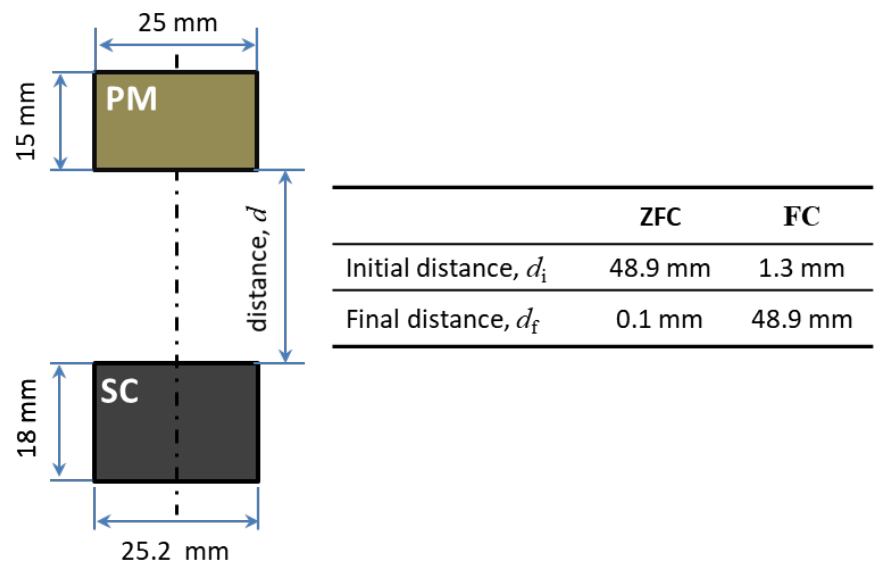

Fig. 2. Arrangement of the PM and the YBCO bulk during the levitation experiments.

\section{B. Zero field cooling}

The levitation force measured in ZFC condition at $75 \mathrm{~K}$ and 
$30 \mathrm{~K}$ is shown in Fig. 3 and Fig. 4 respectively. Four consecutive approaching and leaving cycles of the magnet with respect to the superconductor are shown. Per each cycle, data referring to the descending run (approaching) and the ascending run (leaving) are shown in different style. As it can be seen from the comparison of Fig. 3 and Fig. 4, a higher peak of the levitation force (corresponding to the minimum distance of 0.1 $\mathrm{mm}$ of the magnet from the SC bulk) is obtained at lower temperature, due to the higher critical current of the superconductor. The peak value of the levitation force obtained at the end of each cycle in the range $30 \mathrm{~K}-85 \mathrm{~K}$ is shown in Fig. 5. It can be seen from the figure that the peak levitation force at a given temperature does not significantly change with the cycle. A peak force of only $2 \mathrm{~N}$ is obtained at $85 \mathrm{~K}$. By increasing the temperature, no force was detected (within the sensitivity of the system, which was set to $1 \mathrm{~N}$ ) at $90 \mathrm{~K}$, meaning that the superconducting to normal transition was approached. Based on this observation a critical temperature $T_{\mathrm{c}}$ of about $90 \mathrm{~K}$ can be estimated for the bulk. A large increase of the force can be observed as soon as the temperature decreases below $T_{\text {c. }}$. In fact, a peak force of $85 \mathrm{~N}$ is obtained at $60 \mathrm{~K}$. However, a shallower increase is observed at lower temperatures, and a sort of saturation of the peak levitation force occurs as it can be appreciated from Fig. 5. A similar trend is reported in [21]. This effect is due to the fact that at high critical current density corresponding to low temperature, the maximum possible magnetic moment is induced by the PM in the SC bulk (see section V.A).

We now focus on Fig. 3, reporting the levitation force in ZFC condition at $75 \mathrm{~K}$. Concerning the first cycle, a substantial difference exists between the levitation force obtained during the descending and ascending run. Though reduced, a difference still exists between the force of the descending and ascending run for the other cycles (from $2^{\text {nd }}$ to $4^{\text {th }}$ ). The force of the descending run is higher for the first cycle (sample in the virgin state), whereas it is reduced in the second cycle. This difference is high at high distance of the magnet from the superconductor, and it vanishes at low distance. Starting from the second cycle, the measured data are completely stabilized, which means that the profile of force measured during the descending runs do not change, and so do those measured during the ascending runs (though, the difference of force between ascending and descending runs of one cycle remains). Force behavior outlined above with reference to the temperature of $75 \mathrm{~K}$, also applies at different temperatures, For example, by careful inspection and magnification of Fig. 4, which refers to $30 \mathrm{~K}$, the same behavior can be distinguished. However, by decreasing the temperature the phenomena becomes less evident and the profile of force measured during the subsequent descending and ascending runs become closer. All the phenomenology outlined in this section with reference to the ZFC case, as well as the one discussed in the next section for the FC case, is widely reported in the literature [10], [13], [21], [22]. Same phenomenology also applies for different SC materials, like $\mathrm{MgB}_{2}$ [14], [23].

Due to the fact that a larger levitation force is obtained during the descending run with respect to the ascending run of one cycle, the work spent by the magnet during the approach is higher than the work received by the magnet during the removal. This means that a net mechanical work $L$ is made per cycle by the magnet (supplied by the stepping motors), which can be calculated as the area contained between the descending and ascending force profiles by means of

$L=\oint_{\text {cycle }} F_{z} d z$

The mechanical work per cycle in zero field cooling condition in the temperature range $30 \mathrm{~K}-85 \mathrm{~K}$ is shown in Fig. 6 . We see that the lower the temperature, the lower the separation of the force profiles and the lower the work. The figure also confirms that levitation force cycles are stabilized starting from the second as the same net mechanical work per cycle is obtained. As for the first cycle (virgin sample), since as discussed before a higher repulsive force is obtained during the descending run, a higher work is obtained. It is worth to note that a drop of the work occurs as the temperature of the sample approaches the critical value Tc. This is because at high temperature, the bulk only opposes a weak reaction to the movement of the magnet and both the descending and ascending work vanish.

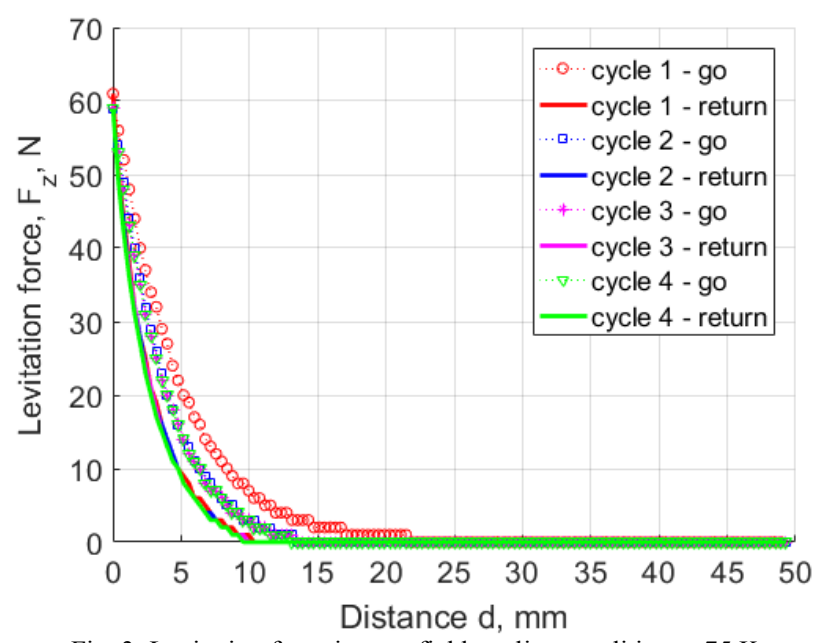

Fig. 3. Levitation force in zero field cooling condition at $75 \mathrm{~K}$. 


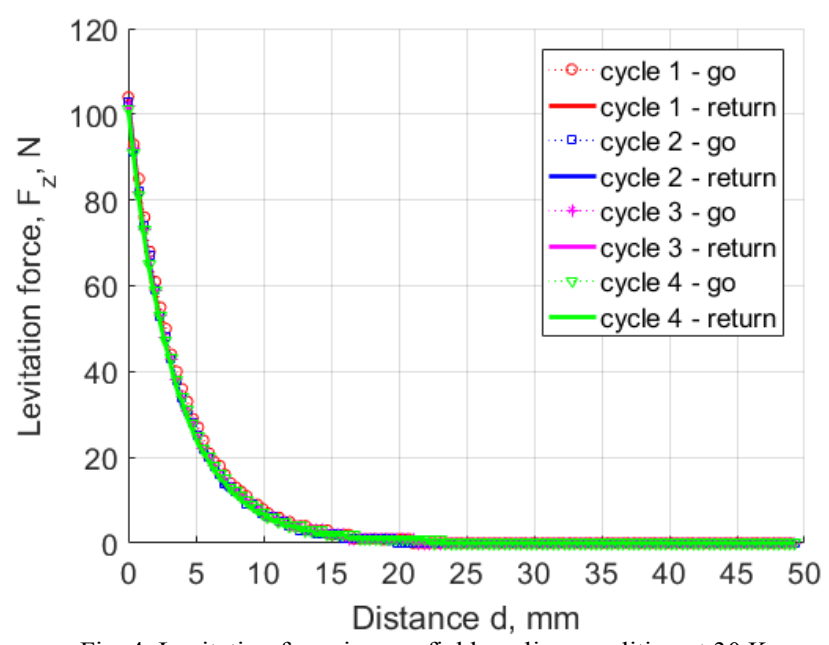

Fig. 4. Levitation force in zero field cooling condition at $30 \mathrm{~K}$.

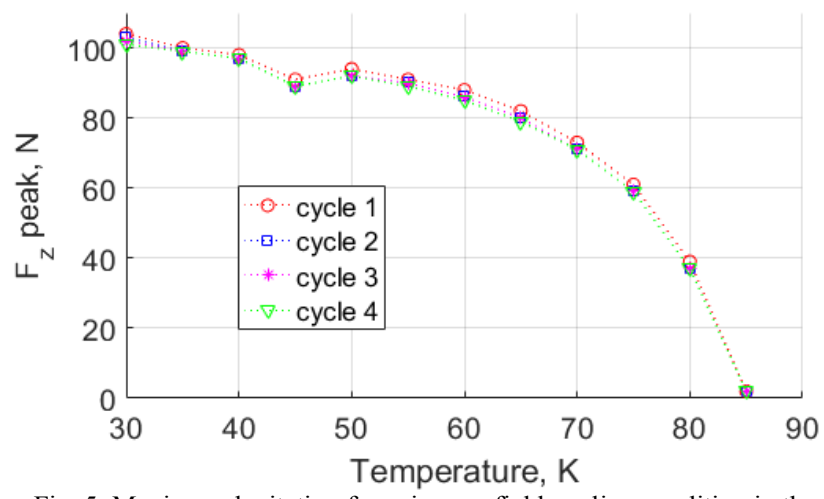

Fig. 5. Maximum levitation force in zero field cooling condition in the temperature range $30 \mathrm{~K}-85 \mathrm{~K}$.

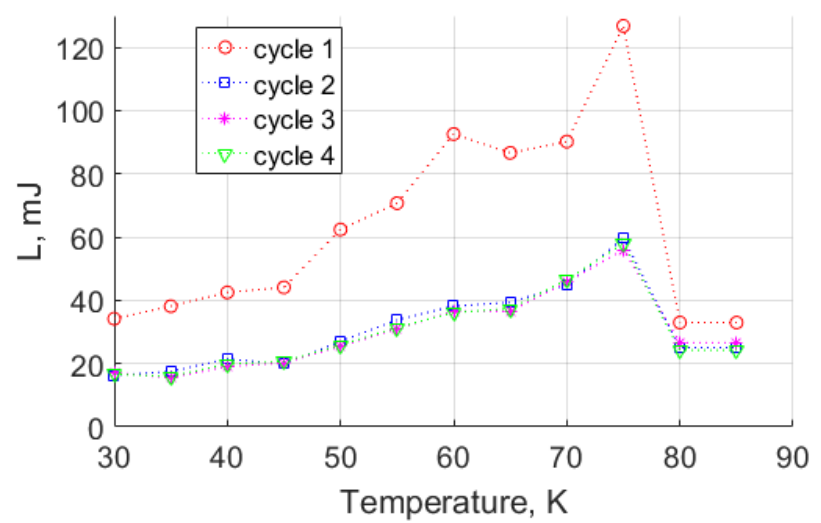

Fig. 6. Mechanical work per cycle in zero field cooling condition in the temperature range $30 \mathrm{~K}-85 \mathrm{~K}$.

\section{Field cooling}

The levitation force measured in $\mathrm{FC}$ condition at $75 \mathrm{~K}$ is shown in Fig. 7. Four consecutive leaving and approaching cycles of the magnet with respect to the superconductor are shown. Per each cycle, data referring to the ascending run (leaving) and the descending run (approaching) are shown in different style. The stepwise change (staircase effect) of the recorded force that is visible in the figure is due to the sensitivity of the force sensor $(1 \mathrm{~N})$. An attractive force is recorded as soon as the magnet starts to move away from the superconductor. A peak of $11 \mathrm{~N}$ is reached at about $5 \mathrm{~mm}$. As the magnet proceeds further the force decreases until it reduces to zero at about $28 \mathrm{~mm}$. As for the descending run, no force is recorded until the magnet returns back to the position where the force reduced to zero during the ascending run (about $28 \mathrm{~mm}$ ). After that, an attractive force is recorded which reaches a peak of $6 \mathrm{~N}$ at about $9 \mathrm{~mm}$. Past this distance the attractive force decreases gradually and it reverses at about $3 \mathrm{~mm}$. A repulsive force of $9 \mathrm{~N}$ is produced by the superconductor on the magnet when this reaches its initial position. During the successive ascending run (second cycle) the force profile follows a different path with respect to the one recorded during for the virgin sample. Return path instead is the same as before. Starting from the second, levitation cycles are almost completely stabilized. As for the case of ZFC, the difference between the force profiles of the ascending and descending runs corresponds to a net mechanical work made per each cycle by the magnet. This work is given by the area contained between the ascending and descending force profiles and can be calculated by means of (1).

The levitation force measured in $\mathrm{FC}$ condition at $30 \mathrm{~K}$ is shown in Fig. 8. The same general behavior as for the data at 75 $\mathrm{K}$ is observed. The main differences are that higher peak values of the attractive force obtained for the ascending and descending runs and the lower value of the repulsive force at the end of the cycle when the magnet recovers the initial position. A further difference is the lower separation between the ascending and the descending profiles, which leads to a lower mechanical work per cycle. The peaks values of the attractive force for the first and the second cycle obtained in the temperature range $30 \mathrm{~K}-85 \mathrm{~K}$ are shown in Fig. 9. The repulsive force measured at the end of the cycles is also reported in the same figure. The net mechanical work made by the magnet per cycle in the same temperature range is shown in Fig. 10.

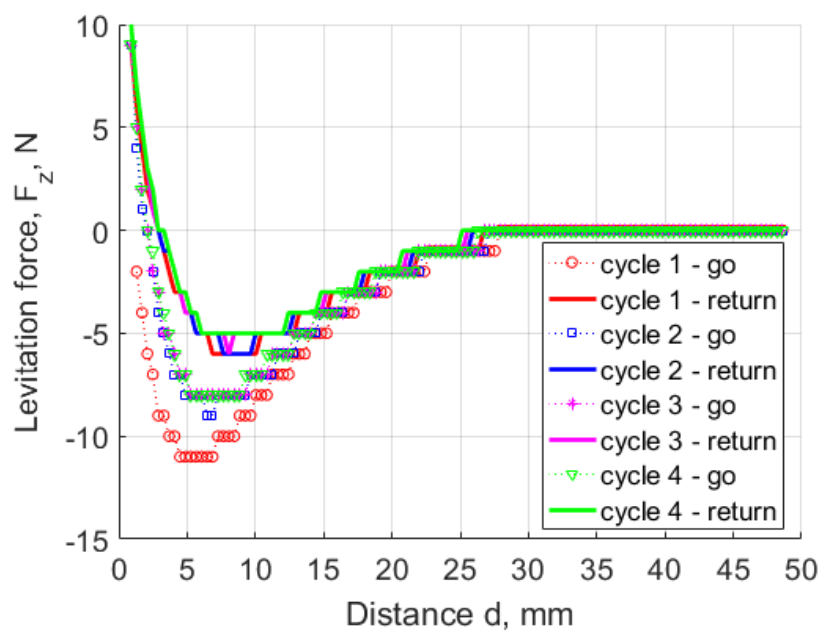

Fig. 7. Levitation force in field cooling condition at $75 \mathrm{~K}$. 


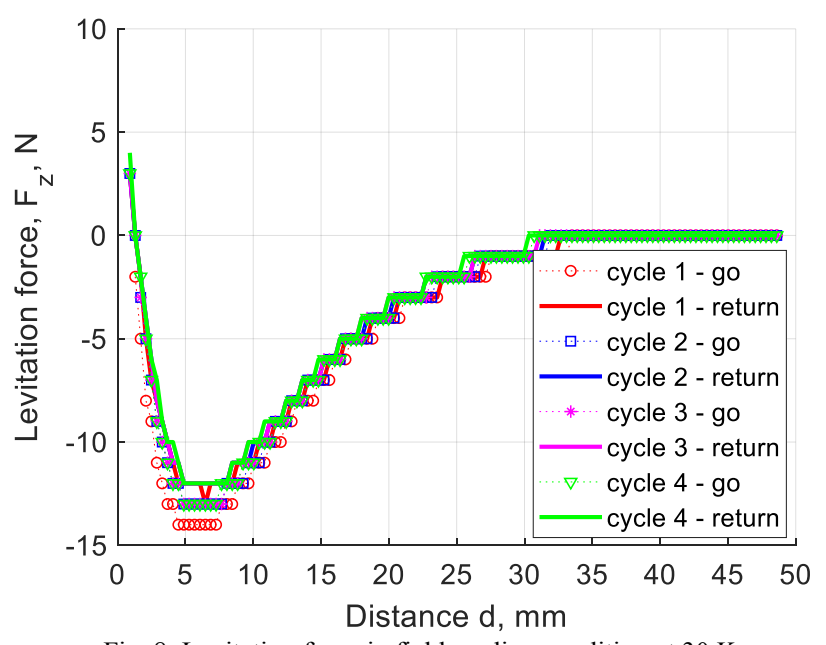

Fig. 8. Levitation force in field cooling condition at $30 \mathrm{~K}$.

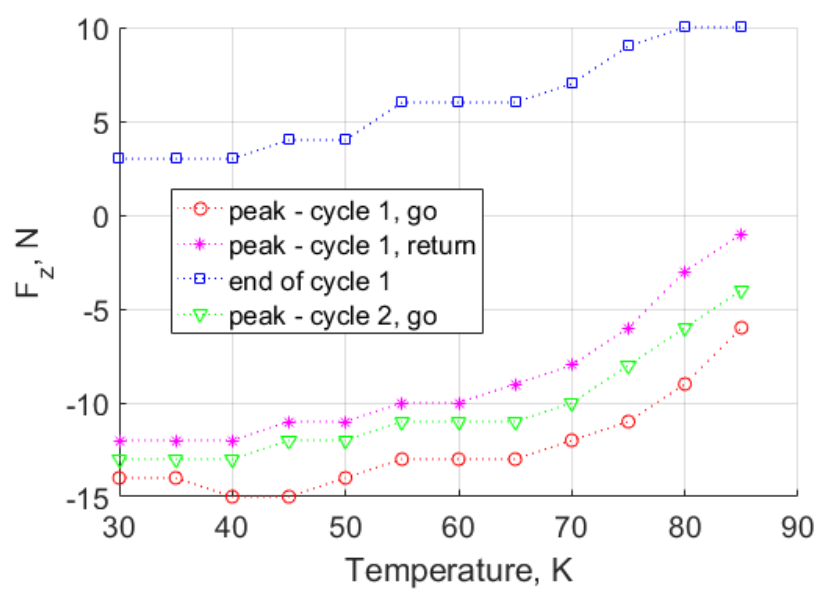

Fig. 9. Peaks of the attractive force (negative) and repulsive (positive) force at the end of the cycle in field cooling condition in the temperature range $30 \mathrm{~K}-85 \mathrm{~K}$.

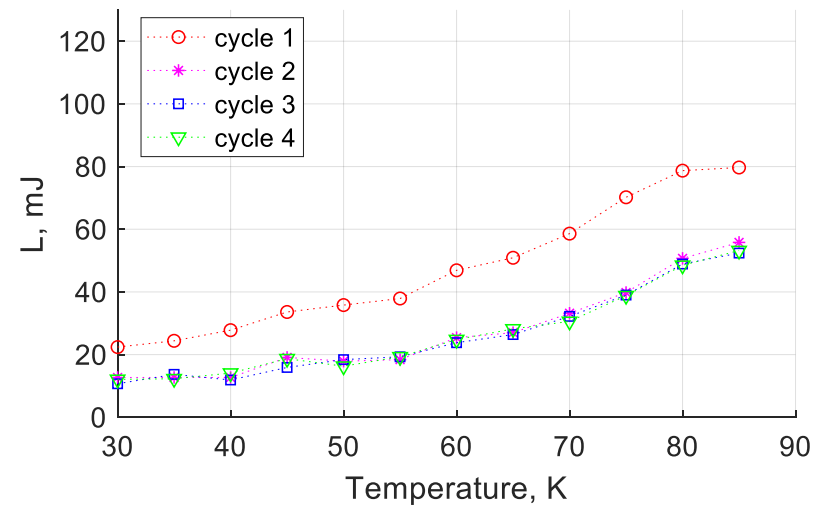

Fig. 10. Mechanical work per cycle in field cooling condition in the temperature range $30 \mathrm{~K}-85 \mathrm{~K}$.

\section{Mathematical Model}

\section{A. Numerical model}

Due to the movement of the PM, currents are induced in the superconductor. These currents interact with the field of the PM via the Lorenz force, thus producing a macroscopic levitation force which can be attractive or repulsive. In order to calculate the induced current and the levitation force a $2 \mathrm{D}$ axisymmetric model based on the volume integral formulation of the eddy current problem is developed. The cylindrical superconductor bulk is subdivided in a finite number of loops with rectangular cross section. Based on the assumption of axial symmetry $\mathbf{J}$ and $\mathbf{E}$ are parallel at any point of the superconductor and oriented along the circumferential direction. The bounded power law is used for modelling the superconductor, i.e. the following expression is assumed for relating the magnitudes of vectors $\mathbf{J}$ and $\mathbf{E}$ [21]-[27]:

$$
\begin{aligned}
& E=\rho(J, B) J \\
& \text { with } \rho(J, B)=\frac{\rho_{\mathrm{SC}}(J, B) \rho_{\mathrm{NS}}}{\rho_{\mathrm{SC}}(J, B)+\rho_{\mathrm{NS}}} \\
& \text { and } \rho_{\mathrm{SC}}(J, B)=\frac{E_{0}}{J_{\mathrm{c}}(B)}\left(\frac{J}{J_{\mathrm{c}}(B)}\right)^{n-1}
\end{aligned}
$$

where $\rho_{\mathrm{NS}}$ is the resistivity of the material in the normal state. The dependence of $J_{\mathrm{c}}$ on $B$ is taken into account. Note that $B$ in (2) is the total magnetic flux density due to both the permanent magnet and the currents induced in the superconductor. The dependence of $J_{\mathrm{c}}$ on $T$ is implicit in (2). Details on the assumed dependence of $J_{\mathrm{c}}$ on $B$ and $T$ are discussed in section V.A. A uniform current density $J$ is assumed for each loop, which is given by the total current $I$ of the loop divided by the cross section $S$ (for the generic loop $k J_{k}=I_{k} / S_{k}$ ). By taking the loop integral of the electric field along the axis of loop $k$ (with radius $r \mathrm{k}$ and cross section $S_{\mathrm{k}}$ ) the following voltage balance is obtained

$$
\begin{aligned}
& R_{k}\left(I_{k}, B_{k}\right) I_{k}=-\frac{d}{d t} \varphi_{k} \quad k=1, \ldots, N \\
& \text { with } R_{k}\left(I_{k}, B_{k}\right)=\rho_{k}\left(I_{k}, B_{k}\right) \frac{2 \pi r_{k}}{S_{k}}
\end{aligned}
$$

where $\varphi_{k}$ is the total flux linked with the loop's axis and $B_{k}$ is the modulus of the field. Equation (3) is obtained by using the Farady's induction law and the constitutive relation (2). The time derivative of the total flux linked with the loop's axis can be expressed as (see the appendix for details):

$$
\frac{d}{d t} \varphi_{k}=\sum_{h} m_{k h} \frac{d}{d t} I_{h}+v \frac{d}{d z} \varphi_{k}^{P M}
$$

where $m_{k h}$ is the mutual induction coefficient between loops $k$ and $h, v$ is the velocity of the permanent magnet and $d \varphi_{k}^{P M} / d z$ is the axial gradient of the flux produced by the permanent magnet on the axis of the loop. By substituting (4) in (3) and by assembling the voltage balance of all loops the following solving system is obtained which allows to calculate the time evolution of the induced currents

$$
\mathbf{M} \frac{d}{d t} \mathbf{I}=-\mathbf{R I}-v \frac{d}{d z} \boldsymbol{\Phi}^{P M}
$$

where $\mathbf{I}$ is the vector of currents of all loops, $\mathbf{M}$ is the full matrix of self/mutual induction coefficients, $\mathbf{R}$ is the diagonal matrix of the resistive voltage drops (dependence of terms of $\mathbf{R}$ on the current and the magnetic field has been kept implicit) and 
$\boldsymbol{\Phi}^{P M}$ is the vector of fluxes produced by the PM. It is important to note that, though not explicitly indicated, vector $\boldsymbol{\Phi}^{P M}$ of fluxes in equation (4) depends on the $z$-position of the PM.

Once the distribution of currents within the superconductor is calculated by solving (5), the total levitation force, which opposes the movement of the PM, may be calculated as

$$
\mathbf{F}=\int_{\tau_{S C}} \mathbf{J} \times \mathbf{B}^{P M} d \tau \Rightarrow\left\{\begin{array}{l}
F_{r}=0 \\
F_{z}=\sum_{k=1}^{N} I_{k} \frac{d}{d z} \varphi_{k}^{P M}
\end{array}\right.
$$

For obtaining (6) formula (A5) of the appendix has been used. Equations (5)-(6) point out that levitation is obtained when a permanent magnet (or more in general a field source) moves along the axial direction provided that the field has a gradient in the same direction.

It is worth to note that in case of an ideal superconductor with unbounded critical current density, the resistive voltage drop ( $\mathbf{R}$ I term) disappears from equation (5). In this case, by solving for I in (5) and by substituting in (6) the following expression of the levitation force produced when the permanent magnet is at position $z$ is obtained

$$
F_{z}(z)=\left(\boldsymbol{\Phi}^{P M}(z)-\boldsymbol{\Phi}^{P M}\left(z_{0}\right)\right)^{\mathrm{t}}[\mathbf{M}]^{-1} \frac{d}{d z} \boldsymbol{\Phi}^{P M}(z)
$$

where $\mathrm{z}_{0}$ is the initial position of the magnet at the moment of the cooling. Equation (7) applies both for the FC and the ZFC case, and independently on if position $z$ is arrived at during the descending or the ascending run. This means that no separation of the descending and ascending runs neither difference between successive cycles can occur in the ideal case. Equation (7) points out that there is a limiting levitation force that can be produced, which only depends on the field profile of the magnet and not on the current density of the superconductor. More precisely, given a certain magnet with assigned field profile and a certain superconductor with assigned current density, a certain force is measured. If the current density is increased (e.g. by decreasing the temperature), a higher force is measured. However, if the current density is increased more the theoretical limit expressed by (7) is reached, and the force does not increase anymore. As expected, this limit value of the force does not depend on the velocity of the PM. Eq. (7) gives a simple algebraic method for calculating the maximum levitation force (no differential equations of the type of (5) must be solved iteratively) and can be used for the fast estimation of the maximum performance and to compare the performance of different arrangement of PMs in ideal condition. The same results are obtained by means of the perfectly trapped flux (PTF) model in [28]-[29].

\section{B. Field and flux of the permanent magnet}

In order to solve the numerical model (5)-(6), the distribution of flux produced by the PM over the HTS bulk must be used as an input. For this purpose, we have measured the magnetic field produced by the PM over a grid of points spanning the volume occupied by the superconductor during the movement of the PM. Measured data at room temperature are shown in Fig. 11. Possible impact of the effective temperature of the PM during the experiments on the field produced are not taken into account. A substantial radial component (and hence a substantial gradient of the flux along the axial direction via equation (A5) of the appendix) can be appreciated close to the lateral borders of the PM. We report that in order to avoid staircase effect on the results due to the large z-spacing of the grid points, we have solved an inverse problem aimed at obtaining a reconstructed distribution of magnetization of the PM able to fit the experimental values at the measurements points. Field data obtained on a finer grid based on the reconstructed magnetization were used as an input in the numerical code. Magnetic fluxes were deduced based on the field data by geometrical processing within the numerical code. We point out that the availability of accurate data of field and flux of the PM is crucial for the accuracy of the numerical results. For this reason, the development of a system for the measurement of the field of PMs with a spatial resolution of 0.1 $\mathrm{mm}$ and at various operating temperatures is in progress at the University of Bologna.

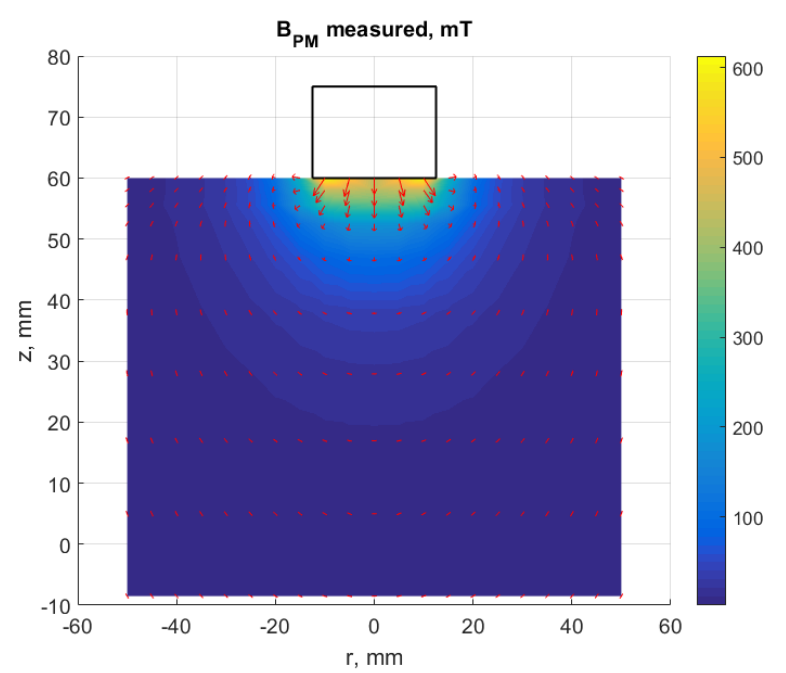

Fig. 11. Measured distribution of magnetic flux density produced by the PM over the volume occupied by the superconductor during the movement

\section{NUMERICAL ANALYSIS AND DISCUSSION}

As discussed in section IV.B the flux density produced by the permanent magnet on the superconductor is in the range $0-0.6$ T. A linear dependence of $J_{\mathrm{c}}$ on $B$ in this range was assumed for calculating the numerical solution. For implementing this dependence in equation (2), at each temperature the values of $J_{\mathrm{c}}$ at $0 \mathrm{~T}$ and at $0.6 \mathrm{~T}$ were assigned and the linear interpolation between these two values was used. Several numerical simulations were carried out and, for each temperature, the values of $J_{\mathrm{c}}$ at $0 \mathrm{~T}$ and at $0.6 \mathrm{~T}$ that allowed the best fitting of the measured levitation force were chosen. Numerical results are presented and discussed in the following. The exponent of the power law used all over the simulations was $n=40$. The normal state resistivity was $3.5 \times 10^{-6} \Omega \mathrm{m}$ [27].

\section{A. Zero field cooling}

The calculated levitation force at $75 \mathrm{~K}$ for the case of zero field cooling during the first cycle is shown in Fig. 12 and 
compared with experiments. The values of $J_{\mathrm{c}}$ at $0 \mathrm{~T}$ and at 0.6 $\mathrm{T}$ used for the calculation are reported in Table II. A good agreement between numerical and experimental data is obtained. Results relative to the subsequent cycles are also in good agreement but they are not reported in the figure for clarity. We report that the numerical profile is rather sensitive to the chosen values of $J_{\mathrm{c}}$. For example if values of Table II are lowered by $30 \%$ a lower peak of the levitation force $(52 \mathrm{~N}$ vs $62 \mathrm{~N}$ ) and a much larger cycle are obtained. Similarly, if values of Table II are increased by $30 \%$ a higher peak of the levitation force $(71 \mathrm{~N}$ vs $62 \mathrm{~N})$ and thinner cycle are obtained. Hence, the numerical model can be used as an effective tool for the estimation of the critical current density of the superconductor in the field range $0-0.6 \mathrm{~T}$. We also report that the values of $J_{\mathrm{c}}$ reported in Table II are in agreement with those obtained with magnetic methods on a similar sample.

In Fig. 13 the calculated distribution of current density and magnetic flux density at points A-D of Fig. 12 are shown. A further point (E), corresponding, as point $\mathrm{A}$, to a distance of 5 $\mathrm{mm}$ of the PM from the bulk but reached during the descending run of the second cycle is also shown in Fig. 13. A lower force $(15 \mathrm{~N}$ vs $21 \mathrm{~N})$ is obtained at point $\mathrm{E}$ than at point A. From Fig. 13 it can be seen that as the magnet moves, currents (indicated with layer 1 in the figure) are induced that impede the diffusion of magnetic field in the bulk. Induced currents create the repulsive levitation force via Lorentz interaction with the field of the magnets. Both current and magnetic field diffuse deeper and deeper as the magnet proceeds. When the magnets reverses the motion after reaching point $\mathrm{B}$, opposite currents (layer 2) are induced that wipe out the previous ones. However, cancellation of the first layer of current induced on the virgin sample is not complete. In fact, as we can see from point $\mathrm{D}$ (end of the cycle) currents of the innermost part of layer 1 are permanently frozen in the superconductor. When the magnet reverses again the motion, moving from point $\mathrm{D}$ toward point E, a further new layer of currents (layer 3 ) is induced that wipes out the previous one (layer 2). Layer 2 is completely wiped out by layer 3 at the end of the descending run of the second cycle. In this position the same distribution of current as for point $B$ is restored. This mechanism of induced current, which steadily repeats during subsequent cycles, is able to explain the observed phenomenology. In particular:

1. The force of the ascending run is always lower than the one of the descending run as currents induced during the return create an attractive effect. The resulting repulsive force is due to the residual part of currents of layer 1 induced during the first descending run. The force decreases as the magnet gets away from the PM since the field becomes weaker and do not interact appreciably with currents.

2. As layer 2 enlarges during the ascending run of the magnet the attractive force it produces becomes larger than the repulsive one produced by the trapped portion of layer 1 . The result is a total attractive (negative) levitation force, which begins at about $10 \mathrm{~mm}$ as it can be seen by careful inspection of Fig. 12. Nevertheless, since this occurs when the magnet is far, the resulting attractive force is weak. The attractive force is not visible in the experimental data of Fig. 12 since it is below the set sensitivity of the force sensor $(1 \mathrm{~N})$. We report that negative force up to $2 \mathrm{~N}$ were measured during the ascending run at higher temperature produced by the deeper penetration of currents. Negative force during the ascending run is also reported by other authors [17], [20], [30]

3. Currents of the descending runs (successive to the first) completely wipe out currents of the ascending runs. The same distribution (as the one of plot B of Fig. 13), and hence the same peak force, is obtained at the end of any descending run.

4. Starting from point B, cycles of induced currents and, consequently, levitation force repeat identically. Differences between the cycles can only occur if appreciable relaxation (associated to low $n$ value) takes place.

Diffusion of current within the superconductor occurs due to the non-unlimited value of the critical current density. In other words, since in magnitude they cannot exceed $J_{\mathrm{c}}$, induced currents occupy more volume in order to produce enough screening capacity to oppose the field of the PM. This diffusion mechanism produces dissipation. In fact, as the magnetic field penetrates deep within the superconductor, the induced electric field becomes non-zero over a finite volume and the loss, calculated as the volume integral of $E J$, becomes non-zero. The instantaneous power loss calculated during the first two cycles in ZFC at $75 \mathrm{~K}$ is shown in Fig. 14. We see that power loss mainly occurs at the middle of the cycle, when the magnet is close and strongly interacts with superconductor. The total energy loss (time integral of the power loss) is also shown in the same figure and compared with the mechanical work done by the PM. The calculated mechanical work during the first cycle is $153.6 \mathrm{~mJ}$, which is about $21 \%$ higher than the experimental value of $126.8 \mathrm{~mJ}$ reported in Fig. 6. This difference is mainly due to the poor resolution of the force sensor which in particular does not allow sensing the attractive (negative) force of about $1 \mathrm{~N}$ occurring during the ascending run, thus leading to underestimation of the actual work.

From Fig. 14. we see that a difference of $31.1 \mathrm{~mJ}$ exists between the mechanical work done by the magnet $(153.6 \mathrm{~mJ})$ and the energy dissipated within the superconductor $(122.5 \mathrm{~mJ})$ during the first cyle. This difference corresponds to the increment of the total magnetic energy of the system. In fact, as it can be seen from Fig. 13.a, a substantial magnetic field is trapped at the top surface of the superconductor when the magnet has moved back to its original position (D). The difference between the mechanical work $(76.0 \mathrm{~mJ})$ and the energy dissipated $(71.1 \mathrm{~mJ})$ reduces to $4.9 \mathrm{~mJ}$ during the second cycle. This means that a greater work is spent during the first cycle in order to produce the magnetic field that is responsible of the levitation force. After that, the work spent is mainly transformed in irreversible power loss, and only marginal change of the magnetic energy occurs due to rearrangement of the currents. 
The calculated levitation force at $30 \mathrm{~K}$ for the case of zero field cooling during the first cycle is shown in Fig. 15 and compared with experiments. The values of $J_{\mathrm{c}}$ at $0 \mathrm{~T}$ and at 0.6 $\mathrm{T}$ used for the calculation are reported in Table II. A good agreement between numerical and experimental data is observed. The levitation force produced by the ideal superconductor with unbounded critical current density, calculated by means of eq. (7), is also shown in Fig. 15. As it can be seen the measured force is very close the ideal value. This means that increased levitation performance of the system can only be obtained if a different arrangement of PM, able to produce a flux distribution with increased axial gradient, is used. No substantial improvement is obtained if a superconductor with higher $J_{\mathrm{c}}$ is used. Equivalently, as shown if Fig. 5, given the layout of the permanent magnet, saturation of the levitation force is obtained by decreasing the operating temperature. We also note that, the higher the $J_{\mathrm{c}}$ the thinner the region of induced currents and magnetic field. Electric field and dissipation hence occur over a smaller volume, thus producing reduced total loss. In the limit of ideal superconductor with infinite $J_{\mathrm{c}}$, surface current distribution is obtained and no dissipation at all is obtained (volume of induced electric field vanishes). This is consistent with the fact that no separation between descending and ascending runs are obtained in the ideal case. Hence, no mechanical work can be done by the magnet and no net loss can be produced in the superconductor.

TABLE II

PARAMETERS OF THE $J_{\mathrm{C}}-B$ DEPENDENCE AND N EXPONENT

\begin{tabular}{cccc}
\hline \hline & Jc at $0 \mathrm{~T}$ & Jc at $0.6 \mathrm{~T}$ & N exponent \\
\hline $75 \mathrm{~K}$ & $1.89 \times 10^{8} \mathrm{~A} / \mathrm{m}^{2}$ & $1.35 \times 10^{8} \mathrm{~A} / \mathrm{m}^{2}$ & 40 \\
$30 \mathrm{~K}$ & $10.95 \times 10^{8} \mathrm{~A} / \mathrm{m}^{2}$ & $8.25 \times 10^{8} \mathrm{~A} / \mathrm{m}^{2}$ & \\
\hline \hline
\end{tabular}

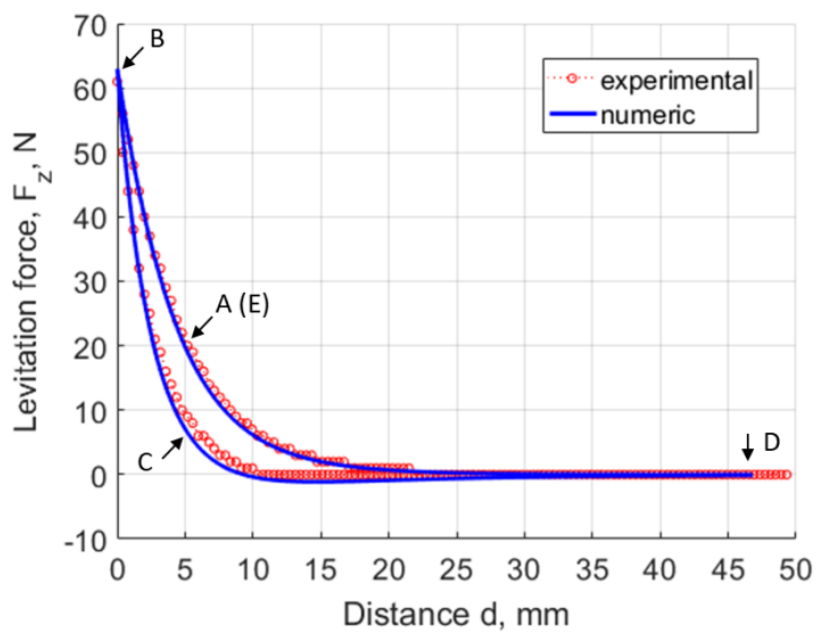

Fig. 12. Numerical and experimental levitation force during the first cycle in zero field cooling condition at $75 \mathrm{~K}$.

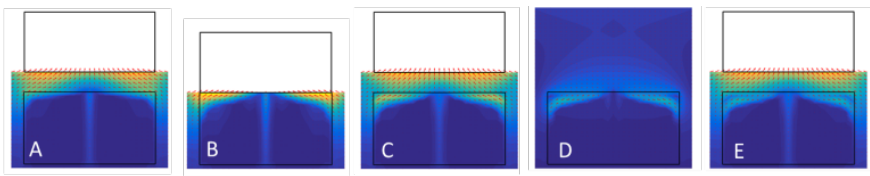

a) Magneti flux density, $T$
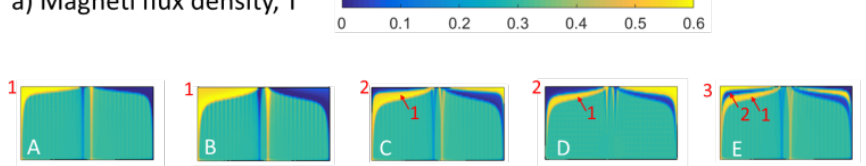

b) Current density, $A / \mathrm{m}^{2}$

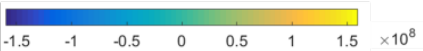

Fig. 13. Induced current and magnetic flux density at $75 \mathrm{~K}$ in ZFC. Points A-E are shown in Fig. 12. Point E belongs to the second cycle.

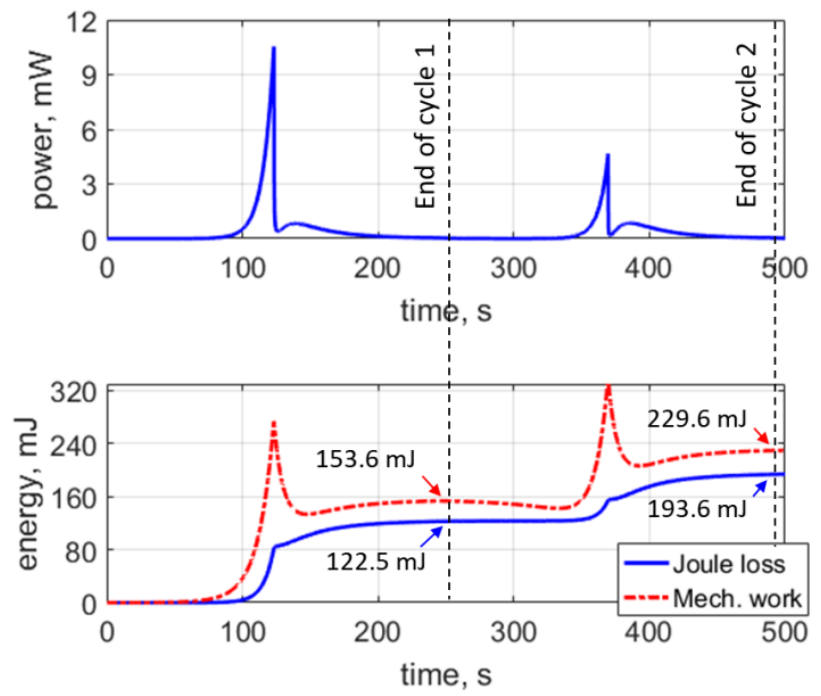

Fig. 14. Instantaneous power loss and total energy loss during the first two cycles in $\mathrm{ZFC}$ at $75 \mathrm{~K}$.

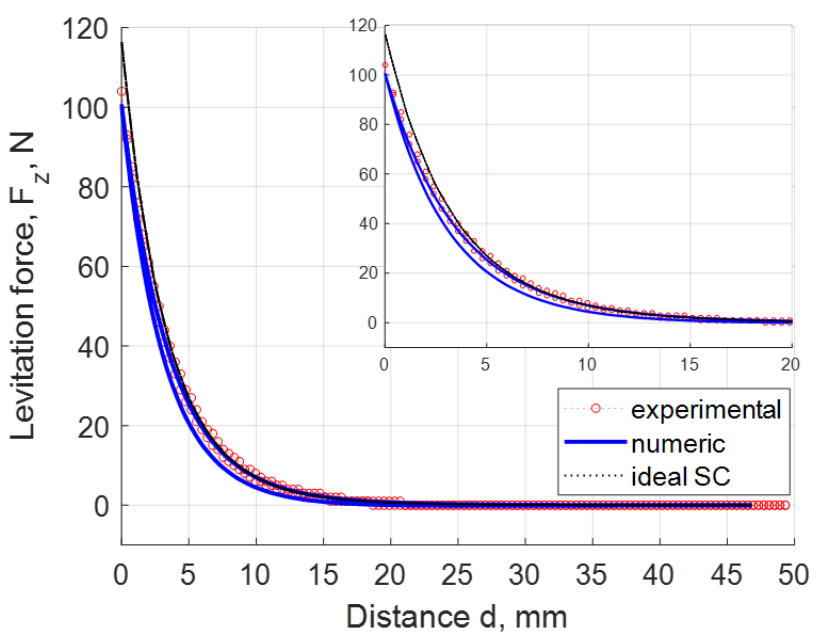

Fig. 15. Comparison between numerical and experimental levitation force during the first cycle in zero field cooling condition at $30 \mathrm{~K}$. The insert shows a zoom on the $\mathrm{x}$-axis interval $[0-20 \mathrm{~mm}]$

\section{B. Field cooling}

The calculated levitation force during the first cycle in FC 
condition is shown in Fig. 16 and compared with experiments. The parameters of the $J_{\mathrm{c}-} B$ dependence used for the calculation are reported in Table II. As for the case of ZFC, a fair agreement is obtained between numerical and experimental results. The levitation force is produced by the Lorentz interaction of the induced currents with the field of the PM. In Fig. 17 the distribution of current density and magnetic flux density corresponding to points A-E of Fig. 16 are shown. As the magnet moves, currents (layer 1) are induced which oppose the decrease of the field in the superconductor and create an attractive levitation force. As the magnet proceeds, more current is induced in the superconductor but the field imposed by the PM is decreased. The competition between these two conflicting effects (the increase of the total induced current and the decrease of the field produced by the PM) produces a maximum of the levitation force, which is reached when the magnet has traveled about $5 \mathrm{~mm}$ (point A). When the magnet reverses the motion after reaching point $\mathrm{B}$, opposite currents (layer 2) are induced that partially wipe out layer 1 and produce a repulsive force. In the first phase of the descending run, the attractive force produced by remaining part of layer 1 still prevails and a total attractive force is obtained. Both the attractive and the repulsive effect increase as the magnet approaches the superconductor. A point is reached however (point C), where the increase of the repulsive effect (which is due to both the enlargement of layer 2 and the increase of the field of the PM) overcomes the one of the attractive effect, and the total force, though still attractive, begins to decrease. At point $\mathrm{D}$, perfect compensation of the attractive and the repulsive force is obtained; no net levitation force is produced. Past point $\mathrm{D}$ the repulsive effect prevails and a total repulsive force, as for example at point $\mathrm{E}$, is obtained. The remaining part of layer 1 visible in plot $\mathrm{E}$ of Fig. 17, becomes permanently frozen in the superconductor. When the magnet reverses again the motion (ascending run of the second cycle) a third layer of current with same direction as layer 1 is induced, which gradually wipes out layer 2. Similarly to the case of ZFC, the portion of layer 2 temporary sandwiched between layer 1 and this third layer is responsible for the lower value of the levitation force obtained during the ascending run of the second cycle.

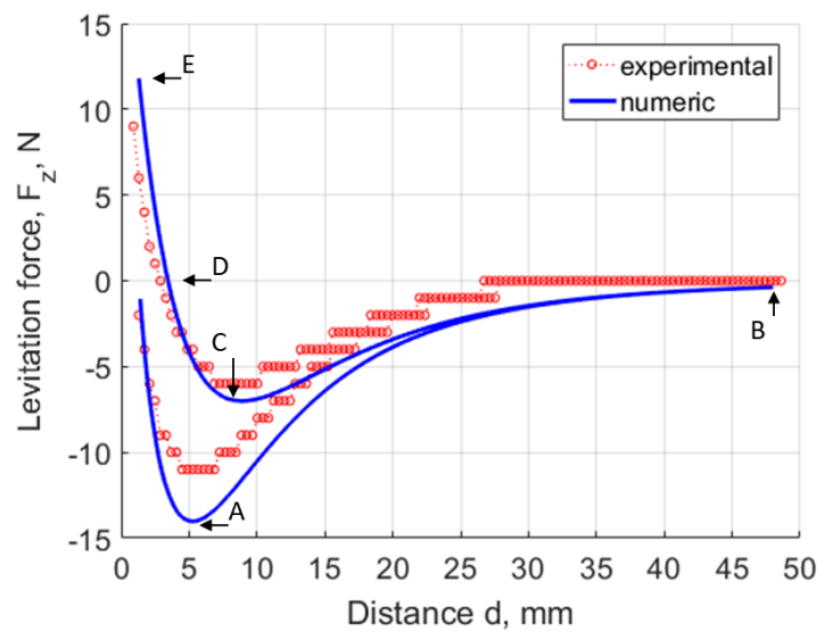

Fig. 16. Numerical and experimental levitation force during the first cycle in field cooling condition at $75 \mathrm{~K}$.

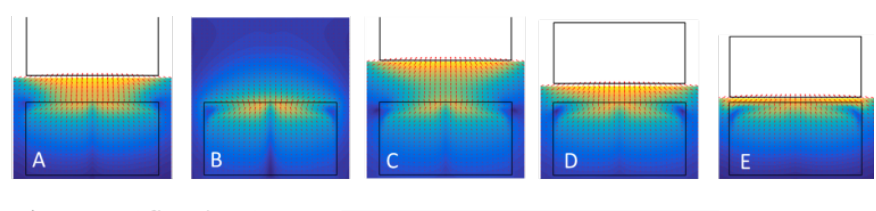

a) Magneti flux density, $T$
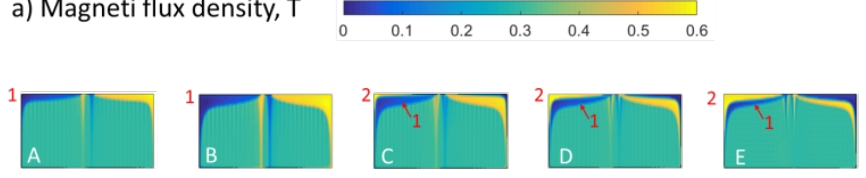

b) Current density, $A / \mathrm{m}^{2}$

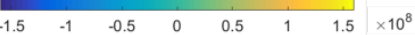

Fig. 17. Induced current and magnetic flux density for FC at $75 \mathrm{~K}$. Points AE are shown in Fig. 16.

\section{CONCLUSION}

An experimental facility for the measurement of the levitation forces between permanent magnets and superconductor samples has been established. The facility is rather general and allows the testing of large superconductor bulks or tape stacks subject to various arrangement of PM over a wide range of temperature. The levitation force between a single grain YBCO cylindrical bulk superconductor and a permanent magnet was investigated by means of the facility, confirming the phenomenology already reported in the literature and outlining the high performance of the bulk. A 2D numerical model was also introduced with the aim to obtain the critical current density of the bulk (in the field range applied by the PM, that is $0-0.6 \mathrm{~T}$ ) by means of the fitting of the measured force. Obtained values of $J_{\mathrm{c}}$ were in agreement with those obtained with magnetic methods on a similar sample. The numerical model also provided a fast way for estimating the performance of the superconductor bulk under ideal condition (with infinite $J_{\mathrm{c}}$ ) and hence for determining the limit value of the levitation force which can be obtained by decreasing the operating temperature of the bulk. Energy loss during one ascending and descending cycle were also analyzed by means of the numerical model, and put in relation with the mechanical work made by the PM. It was shown that a larger work was done by the PM during the first in order to set up the magnetic energy associated to the field trapped by the superconductor.

\section{APPENDIX I}

The general expression of the time derivative of the flux linked with a line $\gamma$ is given by [31]

$$
\frac{d \varphi}{d t}=\int_{\Gamma} \frac{\partial \mathbf{B}}{\partial t} \cdot \mathbf{n} d S-\oint_{\gamma}(\mathbf{v} \times \mathbf{B}) \cdot \mathbf{t} d \gamma
$$

where $\mathbf{v}$ is the velocity of line $\gamma$ with respect to the source of field $\mathbf{B}$. We now consider the 2D axisymmetric problem introduced in section IV.A, made of a superconductor cylinder subject to the field produced by a coaxial permanent magnet moving with velocity $v$ along the axis. The total field $\mathbf{B}$ at any point of the superconductor can be expressed as the sum of the 
field $\mathbf{B}^{\mathrm{PM}}$ produced by the permanent magnet and the field $\mathbf{B}^{\mathrm{J}}$ produced by the current induced in the superconductor, that is

$$
\mathbf{B}=\mathbf{B}^{\mathrm{PM}}+\mathbf{B}^{\mathrm{J}}
$$

The superconductor is subdivided in a finite number of loops. By applying eq. A1 to the axis $\gamma_{\mathrm{k}}$ of loop $k$ and by considering eq. A2 we obtain

$$
\frac{d \varphi_{k}}{d t}=\frac{d}{d t} \int_{\Gamma_{k}} \mathbf{B}^{\mathrm{J}} \cdot \mathbf{n} d S-2 \pi r_{k} v B_{r k}^{P M}
$$

where $\varphi_{k}$ is the total flux linked with the loop's axis and $B_{r k}^{P M}$ is the radial component of the field produced by the PM on the loop's axis. The first term at the right hand side of (A3) is the time derivative of the flux linked with loop $k$ and produced by the currents of all loops. By introducing the magnetic vector potential $\mathbf{A}^{\mathrm{J}}\left(\mathbf{B}^{\mathrm{J}}=\nabla \times \mathbf{A}^{\mathrm{J}}\right)$ this term can be expressed as

$$
\frac{d}{d t} \int_{\Gamma_{k}} \mathbf{B}^{\mathrm{J}} \cdot \mathbf{n} d S=\frac{d}{d t} \int_{\gamma_{k}} \mathbf{A}^{\mathrm{J}} \cdot \mathbf{t} d \gamma=\sum_{h} m_{k h} \frac{d}{d t} I_{h}
$$

where $m_{k h}$ is the mutual induction coefficient between loops $k$ and $h$. Formulae for the calculation of coefficients $m_{k h}$ be found, e.g, in [26], [32]. The following identity holds relating the axial gradient $d \varphi_{k}^{P M} / d z$ of the flux and the radial field $B_{r k}^{P M}$ produced by the permanent magnet on the axis of the loop:

$$
d \varphi_{k}^{P M} / d z=-2 \pi r_{k} B_{r k}^{P M}
$$

Equation (A5) is obtained from the flux balance of the closed cylindrical surface traced by line $\gamma_{\mathrm{k}}$ during an elementary axial movement $d z$. By substituting (A4)-(A5) in (A3) equation (4) is finally obtained.

\section{REFERENCES}

[1] Schaetzle P, Krabbes G, Stoever G, Fuchs G and Schlaefer D, "Multiseeded melt crystallization of YBCO bulk material for cryogenic applications" 1999, Supercond. Sci. Technol. 1269

[2] Ren H, Xiao L, Jiao Y and Zheng M, "Processing and characterization of YBCO superconductors by top-seeded melt growth method in batch process" 2004, Physica C, 412-414 597

[3] Nariki S, Sakai N, Kita M, Fujikura M, Murakami M and Hirabayashi I, "Advances in enlargement of melt-textured $\mathrm{Gd}-\mathrm{Ba}-\mathrm{Cu}-\mathrm{O}$ ", 2006 , superconductors Supercond. Sci. Technol. 19 S500

[4] Kenfaui, D., Sibeud, P.-F., Louradour, E., Chaud, X., Noudem, J.G., “An effective approach for the development of reliable YBCO bulk cryomagnets with high trapped field performances", 2014, Advanced Functional Materials, 24 (25)

[5] J H Durrell, A R Dennis, J Jaroszynski, M D Ainslie, K G B Palmer, YH Shi, A M Campbell, J Hull, M Strasik, E E Hellstrom, D A Cardwell "A trapped field of $17.6 \mathrm{~T}$ in melt-processed, bulk Gd-Ba-Cu-O reinforced with shrink-fit steel", 2014, Superconductor Science and Technology, Volume 27, Number 8

[6] J. R. Hull and M. Murakami, "Applications of bulk high-temperature Superconductors," in Proceedings of the IEEE, vol. 92, no. 10, pp. 17051718, Oct. 2004.

[7] M Strasik1, J R Hull, J A Mittleider, J F Gonder, P E Johnson, K E McCrary and C R McIver, "An overview of Boeing flywheel energy storage systems with high-temperature superconducting bearings", Superconductor Science and Technology, 2010, Volume 23, Number 3

[8] F N Werfel, U Floegel-Delor, R Rothfeld, T Riedel, B Goebel, D Wippich and $\mathrm{P}$ Schirrmeister, "Superconductor bearings, flywheels and transportation", Supercond. Sci. Technol. 25 (2012) 014007 (16pp)

[9] Y. Miyazaki, K. Mizuno, T. Yamashita, M. Ogata, H. Hasegawa, K. Nagashima, S. Mukoyama, T. Matsuoka, K. Nakao, S. Horiuch, T.
Maeda, H. Shimizu, Development of superconducting magnetic bearing for flywheel energy storage system, Cryogenics, Volume 80, 2016, Pages 234-237

[10] John R. Hull and Ahmet Cansiz, "Vertical and lateral forces between a permanent magnet and a high-temperature superconductor", Journal of Applied Physics 86, 6396 (1999)

[11] Alexander A. Kordyuk, "Magnetic levitation for hard superconductors", Journal of Applied Physics 83, 610 (1998);

[12] You-He Zhou, Xian-Feng Zhao, Dynamical analysis to the levitated systems of high temperature superconductors with hysteresis, Physica C: Superconductivity and its Applications, Volume 442, Issue 1, 2006, Pages $55-62$

[13] Yong Yang, Vertical and lateral forces when a permanent magnet above a superconductor traverses in arbitrary directions, Superconductor Science and Technology, 2008, Volume 21, Number 12

[14] E. Perini, G. Giunchi, M. Geri and A. Morandi, "Experimental and Numerical Investigation of the Levitation Force Between Bulk Permanent Magnet and MgB2 disk", IEEE Transactions on Applied Superconductivity, Vol. 19, No. 3, pp. $2124-2128,2009$

[15] A. Morandi, E. Perini, G. Giunchi and M. Fabbri, "Numerical Analysis and Experimental Measurements of Magnetic Bearings Based on $\mathrm{MgB}_{2}$ Hollow Cylinders", IEEE Transactions on Applied Superconductivity, Vol. 21, No. 3, pp. 1460 - 1463, 2011.

[16] C. Navau, N. Del-Valle, and A. Sanchez "Macroscopic Modeling of Magnetization and Levitation of Hard Type-II Superconductors: The Critical-State Model," IEEE Trans. Appl. Supercond. Vol. 23, no. 1, pp. 8201023- 8201023, Feb. 2013.

[17] Xing-da Wu, Ke-Xi Xu, Yue Cao, Shun-bo Hu, Peng-xiang Zuo, Guandong $\mathrm{Li}$, "Modeling of hysteretic behavior of the levitation force between superconductor and permanent magnet", Physica C: Superconductivity, Volume 486, 2013, pp. 17-22

[18] A. Azzouza, H. Allag, J. P. Yonnet and P. Tixador, "3D New Calculation Principle of Levitation Force Between Permanent Magnet and Hard TypeII Superconductor Using Integral Approach," in IEEE Transactions on Magnetics, 2017, vol. PP, no. 99, pp. 1-1.

[19] F Sass, G G Sotelo, R de Andrade Junior and Frédéric Sirois, "Hformulation for simulating levitation forces acting on HTS bulks and stacks of $2 \mathrm{G}$ coated conductors, Superconductor Science and Technology, Volume 28, Number 12, 2015

[20] P Bernstein, L Colson, L Dupont and J Noudem, "Investigation of the levitation force of field cooled $\mathrm{YBCO}$ and $\mathrm{MgB} 2$ disks as functions of temperature", Supercond. Sci. Technol. 30 (2017) 065007 (8pp)

[21] Jun Zhou, Xing-Yi Zhang, You-He Zhou, Temperature dependence of levitation force and its relaxation in a HTS levitation system, Physica C: Superconductivity, Volume 470, Issues 5-6, 2010, Pages 336-339

[22] Y. Lu, D. He and M. Liu, "Magnetic Force Investigation of High-Tc Superconducting Bulk over Permanent Magnet Railway under Different Lateral Offsets with Experimental Methods," Journal of Modern Physics, Vol. 4 No. 6A, 2013, pp. 24-28.

[23] B. Savaskan, E.T. Koparan, S.B. Güner, S. Celik, E. Yanmaz, The size effect on the magnetic levitation force of $\mathrm{MgB} 2$ bulk superconductors, Cryogenics, Volume 80, Part 1, 2016, Pages 108-114

[24] E. H. Brandt, "Geometric barrier and current string in type II superconductors obtained from continuum electrodynamics", 1999, Phys. Rev. B 593369

[25] J. Duron, F. Grilli, B. Dutoit and S. Stavrev, "Modelling the E-J relation of high-T superconductors in an arbitrary current range", Physica C, 2004, Volume 401, Issues 1-4, 15 January 2004, Pages 231-235

[26] Antonio Morandi, "2D electromagnetic modelling of superconductors", 2012, Superconductor Science and Technology, Volume 25, Number 10

[27] M D Ainslie, D Zhou, H Fujishiro, K Takahashi, Y-H Shi and J H Durrell, "Flux jump-assisted pulsed field magnetisation of high-Jc bulk hightemperature superconductors", 2016, Superconductor Science and Technology, Volume 29, 124004 (9pp)

[28] H. May, R. Palka, E. Portabella and W. Canders, "Evaluation of the magnetic field-high temperature superconductor interactions", 2004, COMPEL, Vol. 23, pp. $286-304$

[29] A. Patel, S. Hahn, J. Voccio, A. Baskys, S. C. Hopkins and B. A. Glowacki, "Magnetic levitation using a stack of high temperature superconducting tape annuli", 2016, Superconductor Science and Technology, Volume 30, Number 2

[30] W. M. Yang, X. X. Chao, X. B. Bian, P. Liu, P. X. Zhang and L. Zhou, "The effect of magnet size on the levitation force and attractive force of single-domain YBCO bulk" superconductors Supercond. Sci. Technol. 16 (2003) 789-792 
[31] J. D. Jackson, “Classical Electrodynamics", $2^{\text {nd }}$ Edition, 1975, John Wiley \& Sons - New York, pp. 209-213

[32] W. G. Hurley, M. C. Duffy, J. Zhang, I. Lope, B. Kunz and W. H. Wölfle, "A Unified Approach to the Calculation of Self- and Mutual-Inductance for Coaxial Coils in Air," in IEEE Transactions on Power Electronics, vol. 30, no. 11, pp. 6155-6162, Nov. 2015. 